\title{
OPEN Reorganization of nurse scheduling reduces the risk of healthcare associated infections
}

\author{
Eugenio Valdano ${ }^{1}$, Chiara Poletto ${ }^{1}$, Pierre-Yves Boëlle ${ }^{1} \&$ Vittoria Colizza ${ }^{1,2} \llbracket$
}

Efficient prevention and control of healthcare associated infections (HAIs) is still an open problem. Using contact data from wearable sensors at a short-stay geriatric ward, we propose a proofof-concept modeling study that reorganizes nurse schedules for efficient infection control. This strategy switches and reassigns nurses' tasks through the optimization of shift timelines, while respecting feasibility constraints and satisfying patient-care requirements. Through a SusceptibleColonized-Susceptible transmission model, we found that schedules reorganization reduced HAI risk by $27 \%$ ( $95 \%$ confidence interval $[24,29] \%$ ) while preserving timeliness, number, and duration of contacts. More than $30 \%$ nurse-nurse contacts should be avoided to achieve an equivalent reduction through simple contact removal. Nurse scheduling can be reorganized to break potential chains of transmission and substantially limit HAI risk, while ensuring the timeliness and quality of healthcare services. This calls for including optimization of nurse scheduling practices in programs for infection control in hospitals.

Healthcare associated infections (HAIs) are increasingly widespread, with an estimated 4 million individuals affected each year in Europe, representing approximately $6 \%$ of all hospitalized patients ${ }^{1}$. These infections have a substantial impact on morbidity and associated costs for the healthcare system, potentially leading to failure of treatment, longer illnesses and hospitalizations, and deaths. Rising antimicrobial resistance in hospitals has also increased the threat to human health, as resistant pathogens may cause serious infections that cannot be treated with available drugs ${ }^{2}$.

Common HAIs spread through close-range proximity or physical contacts between individuals. Several studies highlighted the importance of contacts for HAI diffusion ${ }^{3-9}$, showing how larger variety and duration of contacts are associated to an increase in HAI risk ${ }^{10,11}$. These factors lead to the well-known paradox that healthcare workers play a key role in pathogen dissemination because of their frequent and persistent contacts with individuals of different categories ${ }^{12}$. Being at higher risk for HAI colonization, healthcare workers may act as transient superspreaders and transmit the infection to the large number of individuals they get in contact with, especially in the vulnerable population of patients ${ }^{12-14}$.

Infection control strategies targeting healthcare workers require careful design, to avoid interfering with their ability to carry out their core healthcare responsibilities. Hygienic measures such as hand sanitizing are the primary strategy to prevent HAI diffusion, aiming to reduce the per-contact risk of transmission ${ }^{15}$. The efficacy of these measures is however limited by low compliance rates, as reported by several studies especially under conditions of emergency or understaffing ${ }^{12,16-18}$. Even low compliance by a few individuals can have a disproportionate impact on the risk of HAI diffusion in the hospital, given the presence of potential superspreaders ${ }^{11,12,19,20}$. Other approaches for infection control have therefore considered the use of personal protective equipment (e.g. face masks and gloves) ${ }^{21}$, vaccination ${ }^{22}$, isolation, or nurse cohorting (i.e. assigning nurses to a limited number of patients during a given working period) ${ }^{23}$. Their effectiveness, however, is still matter of debate ${ }^{23,24}$. Most importantly, some of these measures may only be applicable in reaction to outbreaks, as they are rather costly and disruptive. It may thus prove difficult to integrate them into day-to-day hospital activities.

Routine operations in a hospital are ensured by adequate healthcare workers staffing and scheduling. Their organization has been extensively studied for several decades in operations research, management, and computer science ${ }^{25,26}$ and is generally known as the 'nurse scheduling problem'. It typically involves the optimization of single or multiple goals while satisfying a set of hard constraints-i.e. features that need to be respected at all costs, e.g. feasibility, workload, length of shifts, required personnel or skills—and a set of soft constraints-i.e. aspects

${ }^{1}$ INSERM, Sorbonne Université, Institut Pierre Louis d'Epidémiologie et de Santé Publique, IPLESP, 27 rue Chaligny, 75012 Paris, France. ${ }^{2}$ Tokyo Tech World Research Hub Initiative, Institute of Innovative Research, Tokyo Institute of Technology, Tokyo, Japan. ${ }^{\square}$ email: vittoria.colizza@inserm.fr 

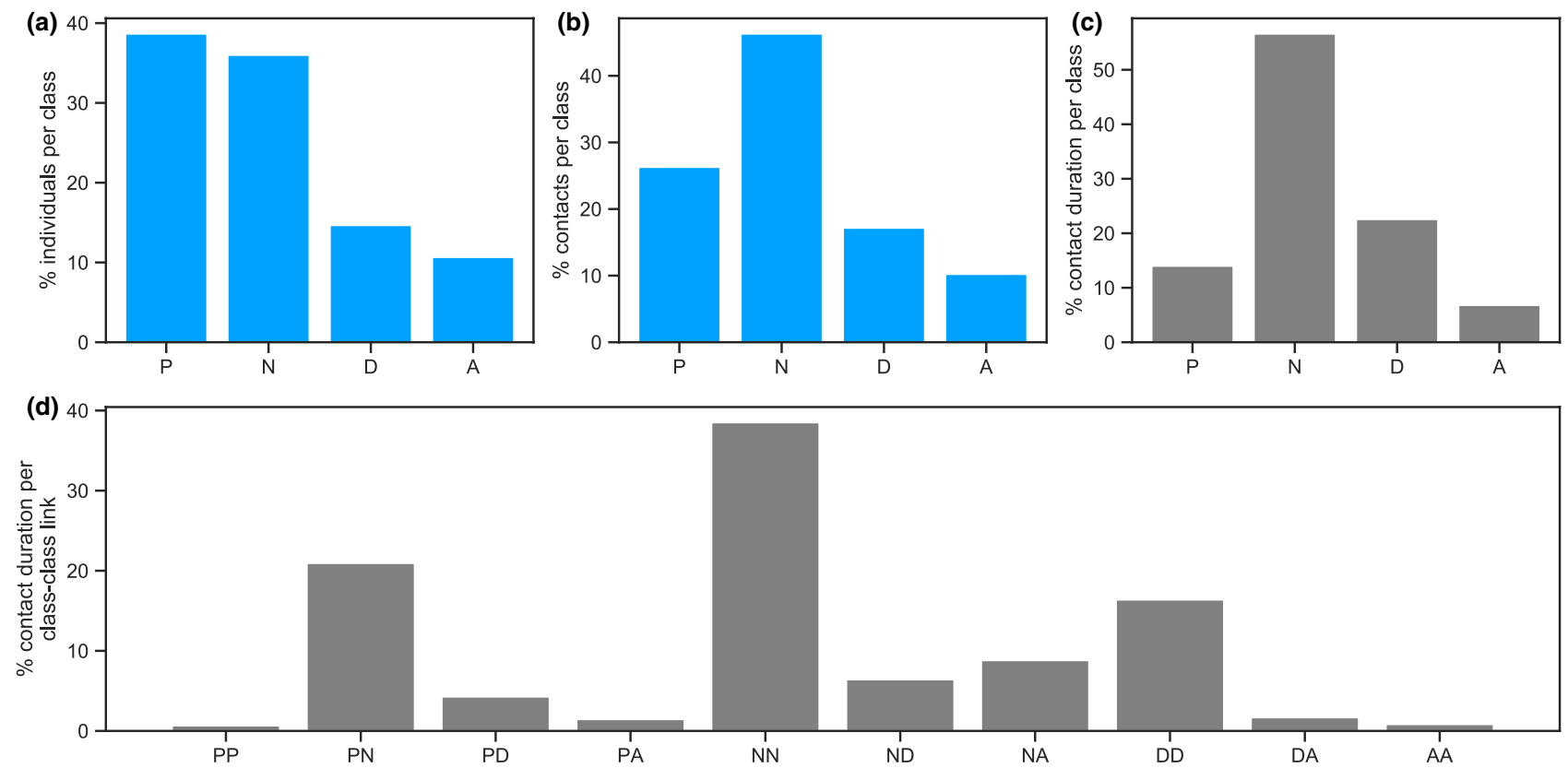

Figure 1. Contacts in the hospital ward. Percentage of participating individuals (a), of contacts (b), and of contact duration (c) by class of individuals [patients (P), nurses (N), doctors (D), administrative personnel (A)]. (d) Percentage of contact duration between classes of individuals.

that are desirable but may not be met in order to achieve a solution, e.g. preferences for a day off. Mathematically described by the constrained minimization of a potential function, the solution to the scheduling problem aims to optimize human resources' efficiency, patient safety, quality of medical services, costs, and staff satisfaction. Despite the great interest in the topic, research has so far addressed it exclusively from the management and computational perspectives ${ }^{25,26}$, with no regards to its potential role in infection control.

Here we propose a proof-of-concept modeling study for hospital infection control based on the reorganization of care in a hospital ward through changes in the schedule of work shifts of nurses. Using high-resolution temporal records on contacts in a hospital ward ${ }^{3}$, our approach switches tasks between nurses by altering their work schedules through the optimization of a potential function, similarly to models for nurse scheduling. The reorganized schedule maintains full staff capacity at any given time, preserves all time-referenced contacts recorded in the dataset without affecting quality standards of medical services, and respects basic occupational constraints (weekly workload, length of a work shift). The study is applied to a short-stay geriatric hospital ward in Lyon, France, where contact data were collected through automated sensors ${ }^{3}$. We model the circulation dynamics of hand-transmitted pathogens such as methicillin-resistant Staphylococcus aureus (MRSA) or vancomycinresistant Enterococci (VRE) in the ward, and evaluate the effectiveness of the intervention by measuring the risk for HAI diffusion.

\section{Methods}

Contact data. We used publicly available anonymized data collected during 4 days and 4 nights (December 6 to 10,2010$)$ at a short-stay geriatric ward of a hospital in Lyon, France ${ }^{3,27}$. Using wearable RFID sensors, the system tracked face-to-face proximity contacts over time between 75 participating individuals, including 27 nurses (N), 11 doctors (D), 8 administrative staff (A), and 29 patients (P). The dataset was first analyzed in Ref.'; Fig. 1 reports its basic properties. Nurses and doctors had the largest cumulative duration of contacts, and most frequent contacts were observed between nurses (NN), and between patients and nurses (PN). The Supplementary Information reports additional information on data collection and characteristics.

Ethics statement. The contact data used in this study were collected and made publicly available ${ }^{3,27}$ by a research study approved by the French national bodies responsible for ethics and privacy, the "Commission Nationale de l'Informatique et des Libertés" (CNIL, http://www.cnil.fr) and the "Comité de Protection des personnes" (http://www.cppsudest2.fr/) of the hospital.

HAl risk estimate. The time-resolved contacts are represented in the form of a temporal contact network ${ }^{28}$, where nodes correspond to individuals and links to proximity encounters. Time evolution occurs at an hourly timescale. We model HAI diffusion in the hospital ward through a Susceptible-Colonized-Susceptible transmission dynamics on the temporal contact network ${ }^{6,8,19,29,30}$. Colonized individuals transmit the pathogen with probability $\lambda$ per contact. Their average colonization duration is fixed at $10 \mathrm{~h}$ for healthcare workers, assuming a spontaneously clearing transient colonization at the end of a work shift. The duration is longer for patients and 
(a)

EMPIRICAL

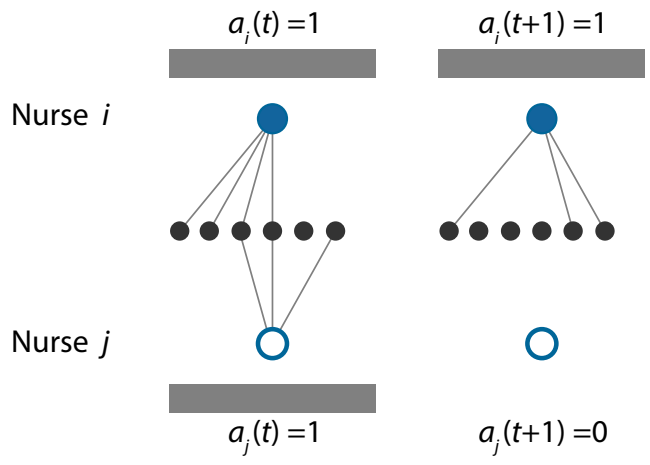

REORGANIZED

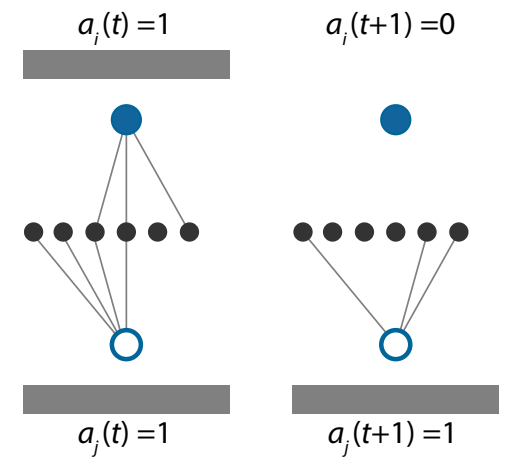

(b)

\begin{tabular}{rl|lll|l|l|l|l|l|l|} 
EMPIRICAL \\
REG (-1)
\end{tabular}

Figure 2. Intervention on nurse scheduling. (a) Schematic visualization of the intervention, with the exchange of tasks between nurse $i$ (filled blue node) and nurse $j$ (void blue node), at times $t$ (while they are both at work) and $t+1$ (while nurse $j$ is not at work in the empirical schedule, and would exchange her shift with nurse $i$ in the reorganized schedule). Links represent contacts with other individuals (black nodes). The reorganized nurse schedule (right) is compared to the empirical one (left) obtained from the contact data. (b) Example of an empirical nurse schedule along with the re-organized ones obtained with $k=-1$ and $k=1$, leading to regular and irregular individual schedules, respectively. Grey blocks correspond to hours when the nurse is at work.

corresponds to 10 days, hypothesizing a weekly bacterial screening, followed by 3 days to obtain test results and implement a decolonization therapy, as in Refs. ${ }^{6,19}$.

To assess the risk of transmission of the infection in the ward, we estimate the condition for circulation of MRSA or VRE on measured contacts through the infection propagator approach ${ }^{31-33}$. This theoretical framework was introduced to study epidemics spreading on temporal networks and identify the critical value $\lambda_{c}$ of the transmissibility above which the pathogen spreads in the host population (i.e. if $\lambda>\lambda_{c}$ an outbreak is predicted to occur). The Supplementary Information reports a full description of this approach, and the available software tool.

Intervention through isolation or contact removal. To assess the role that each class of individuals has on HAI risk, we simulate two interventions based (i) on the isolation of individuals belonging to a given class, and (ii) on the removal of contacts established between two classes (e.g. contacts between patients and nurses). They both mimic emergency outbreak responses, as they potentially disrupt ward operations. The former reproduces quarantining a subset of individuals, either-if they are patients or staff-to prevent them from spreading the infection, or-in the case of patients-to protect them from acquiring the infection. The intervention by removal of contacts established between two classes reproduces restricting allowed contacts among classes to the minimum necessary to provide essential or critical care. Each intervention is made comparable across classes or pairs of classes, through the isolation of 8 individuals (i.e. the smallest size class) or the removal of $5 \%$ of the total duration of contacts in the dataset, respectively. Interventions are repeated to account for the stochasticity in the choice of the node to isolate or of the contacts to remove.

Intervention based on reorganization of nurse scheduling. We introduce an activity variable $a_{i}(t)$ associated to nurse $i$ during hour $t$, so that $a_{i}(t)=1$ if the nurse is at work and establishes contacts in that hour and $a_{i}(t)=0$ otherwise (Fig. 2a). For each nurse, we compute the shift duration $s$ defined as the number of consecutive work hours, and the workload $w$ corresponding to the total number of hours worked in the dataset.

The proposed intervention switches and reassigns the tasks performed by two nurses in a given hour $t$. Tasks consist in contacts that nurses establish, as they perform their duties in interaction with other individuals 
(e.g. caring for a patient). They correspond to possible transmission events. The reorganization is driven by the minimization of the following potential function:

$$
V=-\frac{k}{2} \sum_{i} \sum_{t_{1}} \sum_{t_{2}} a_{i}\left(t_{1}\right) a_{i}\left(t_{2}\right)\left(t_{1}-t_{2}\right)^{2} .
$$

$i$ runs on all nurses, $t_{1}$ and $t_{2}$ run on the whole timeline, and $k$ determines the tendency of the potential $(k= \pm 1)$. This generates the following dynamics. If $k$ is negative, and a nurse is active during hour $t$, minimizing the potential will increase the probability that the nurse is active also immediately before or after $t$. If $k$ is positive, and a nurse is active during hour $t$, minimizing the potential will increase the probability that the nurse is active also at times much before, or much after $t$. In addition, $k=-1$ shows a tendency to create regular individual schedules (periodic activity patterns), and $k=1$ a tendency for irregular individual schedules (erratic activity patterns) (Fig. 2b). Schedules are regular if individual work shifts and breaks tend to have constant, or periodical (e.g., weekly patterns) durations. These are typical of routine hospital operations in nonemergency conditions. Schedules are irregular if instead individual shifts and breaks have variable durations and do not follow routine patterns. These may represent the temporary recruitment of part-time personnel in time of crisis.

This strategy preserves the number, type, and exact timeline of contacts, differently from the intervention through contact removals. The minimization of the potential is additionally subject to feasibility constraints on shift duration and workload of nurses:

- Model $S$ : the exchange is allowed as long as each working shift lasts at most $s=10 \mathrm{~h}$, as measured empirically.

- Model WS: in addition to the constraint on shift duration, the exchange is allowed only if it preserves the empirically measured workload $w$ of each nurse.

Each model is run with both values of $k$, for a total of 4 reorganization options $\left(S_{-1}, S_{+1}, W S_{-1}, W S_{+1}\right)$. The Supplementary Information reports a detailed description of the minimization algorithms. Despite being synthetic, these interventions have an increasing degree of realism to show the potential of this proof-of-concept study for possible applications in real situations.

Evaluation of interventions. We evaluate the effect of interventions by comparing the resulting HAI risk estimate $\left(\lambda_{c}^{I N T}\right)$ with the one estimated on the empirical pattern of contacts $\left(\lambda_{c}^{E M P}\right)$. We define the HAI risk reduction as the relative variation of these two quantities $\left(\left(\lambda_{c}^{I N T}-\lambda_{c}^{E M P}\right) / \lambda_{c}^{E M P}\right)$, so that a positive risk reduction corresponds to interventions improving the control of potential HAI diffusion in the hospital ward (the opposite for negative values). Fluctuations in the HAI risk reduction are obtained from the variations resulting from the stochastic trials.

Effects of the reorganization of nurse scheduling on contact patterns. To test whether the proposed reorganization of nurse scheduling leads to nurse cohorting ${ }^{23}$, we measure the variation in the number of distinct nurses assigned to each patient following the intervention compared to the empirical value. Negative values of this variation correspond to nurse cohorting (i.e. an average reduction of the number of nurses per patient).

We also measure the variations in the nurses' degree (i.e. number of distinct connections each nurse establishes) by comparing average degree and associated fluctuations before and after the reorganization.

\section{Results}

Intervention through isolation or contact removal. Complete isolation of 8 randomly chosen patients corresponds to a drop of $8 \%$ in the cumulated duration of contacts, while isolating 8 nurses reduces it by $31 \%$ (Fig. 3a). In the latter situation, it leads to an approximate $35 \%$ median reduction of the HAI risk (Fig. 3c), whereas the same intervention applied to other classes has negligible impact.

When removing a certain fraction of contacts between classes, the largest risk reduction is obtained by acting on nurse-nurse contacts (median reduction of 13\%), corresponding to deleting $15 \%$ of nurse-nurse contact duration (Fig. 3b,d). Interventions on contacts between nurses and doctors or administrative staff, which are proportionally more disruptive, have almost no impact on the risk.

Both theoretical interventions highlight the central role played by nurses in the hospital ward under study, supporting the design of a more realistic intervention that could act on nurse activities without disrupting the ward functioning and the provision of medical and nursing services.

Intervention based on reorganization of nurse scheduling. Minimizing the potential while constraining only the maximum shift duration leads to two different profiles of the workload distribution (Fig. 4). Model $S_{-1}$ shows approximately half of the nurses not working $(w=0)$, and the rest distributed quite evenly from short to very long workloads (Fig. 4a). Model $S_{+1}$ tends instead to homogenize nurses' workload around the average value (16-23 $\mathrm{h}$ in the 4 -day timeframe, Fig. $4 \mathrm{c}$ ).

Shift duration distributions are rather similar in all models, and qualitatively comparable with empirical data (Figs. 4 and 5). Small variations on 1-h shifts (higher probability in WS models, Fig. 5b,d) and 8-9-h shifts (more marked increase in the $S$ models, Fig. 4 b,d) are observed.

Model $S_{+1}$ achieves the largest reduction of HAI risk (median $27 \%$ reduction, $95 \%$ CI $[24,29] \%$ ), followed by $W S_{-1}(21 \%,[20,24] \%)$ and $W S_{+1}(19 \%,[16,20] \%)$ (Fig. 6a). Equivalent risk reductions would be obtained 

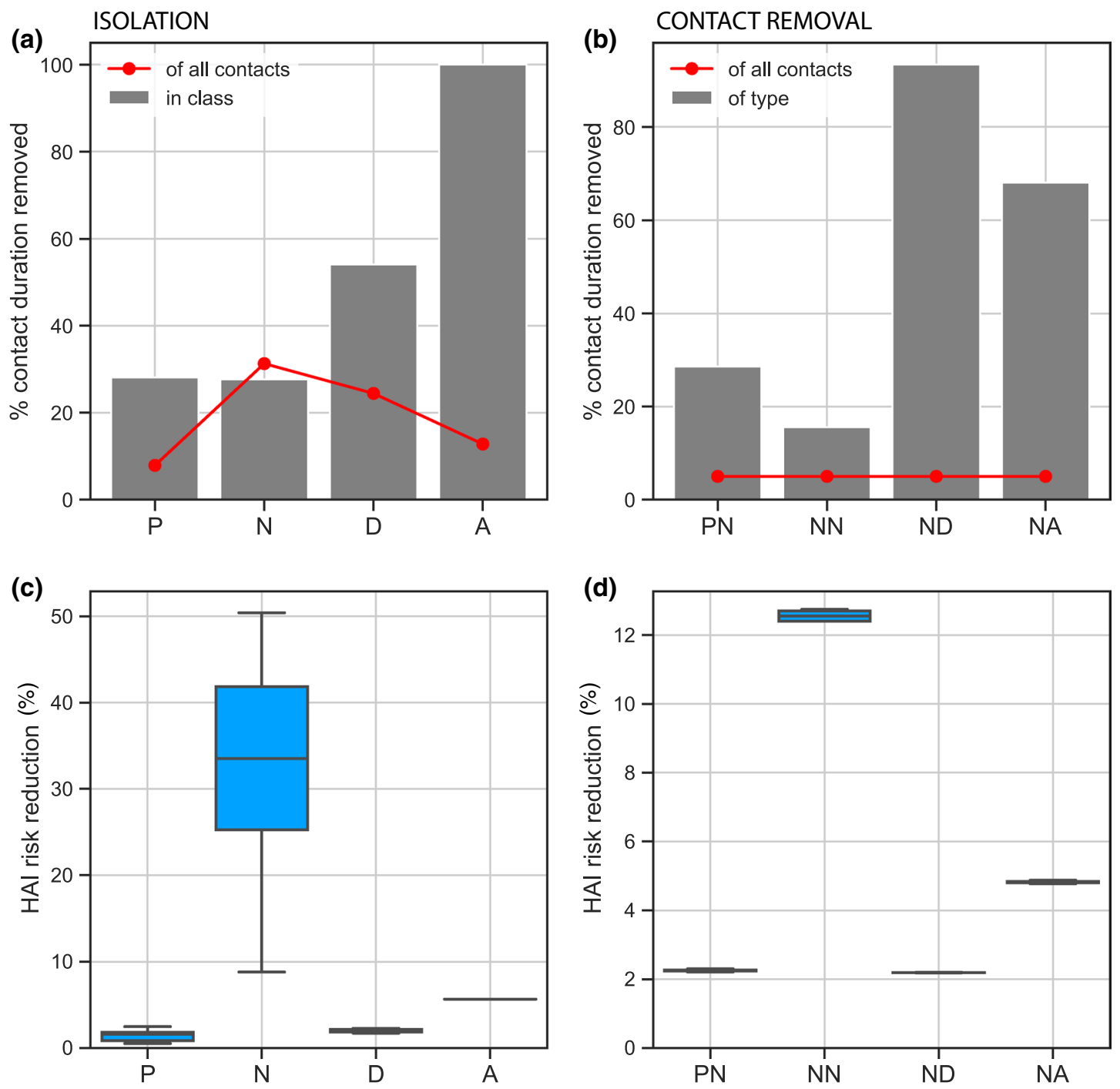

Figure 3. Impact of intervention through isolation or contact removals. (a) Median percentage of contact duration removed within the class (red line) or of the full timeline (grey bars) once 8 individuals in each class are isolated, corresponding to $28 \%$ of patients $(\mathrm{P}), 30 \%$ of nurses $(\mathrm{N}), 73 \%$ of doctors $(\mathrm{D})$, and $100 \%$ of administrative staff (A). (b) Median percentage of contact duration removed among links established by nurses with other classes [grey bars, with patients $(\mathrm{PN})$, with nurses $(\mathrm{NN})$, with doctors $(\mathrm{ND})$, with administrative staff (NA)], once $5 \%$ of the total contact duration of the full timeline is removed (red line). (c,d) HAI risk reduction in the hospital ward achieved through isolation (c) or contact removal (d) corresponding to the results of panels (a) and (b), respectively. Boxplots indicate the median, interquartile range and 95\% CI of the risk reduction, accounting for the stochasticity of the interventions (results from 20 random trials).

by contact removal if more than $30 \%, 25 \%$, and $20 \%$ duration of nurse-nurse contacts were to be removed, respectively (Fig. 6b). Model $S_{-1}$ instead increases HAI risk of $5 \%$.

Models $S_{+1}, W S_{-1}$, and $W S_{+1}$, which decrease risk, show a reduction of the fluctuations in the number of distinct contacts established by nurses, without substantially altering their average number of contacts or the number of distinct nurses assigned to each patient (Fig. 7). Model $S_{-1}$, which increases risk, raises cohorting levels with a median of 4 less nurses assigned to each patient and strongly increases nurses' degree fluctuations.

\section{Discussion}

The key role of healthcare workers in the transmission of healthcare associated infections is widely recognized ${ }^{12-15}$. Low compliance and limited sustainability of recommended strategies hinder efficient infection control. Our study proposes an alternative change of practice through the reorganization of nurse work shifts to reduce HAI risk. Using sensed contact data in a hospital ward, we show that reassigning tasks to nurses minimizing a potential function on their timeline of activity can reduce the risk of HAI diffusion by about one third. Our findings show the potential of planning nurse schedules to improve infection prevention and control.

The key advantage of the proposed intervention is that it preserves the number, type, and duration of contacts at each time. This ensures the timeliness and quality of medical and nursing services provided. An equivalent 

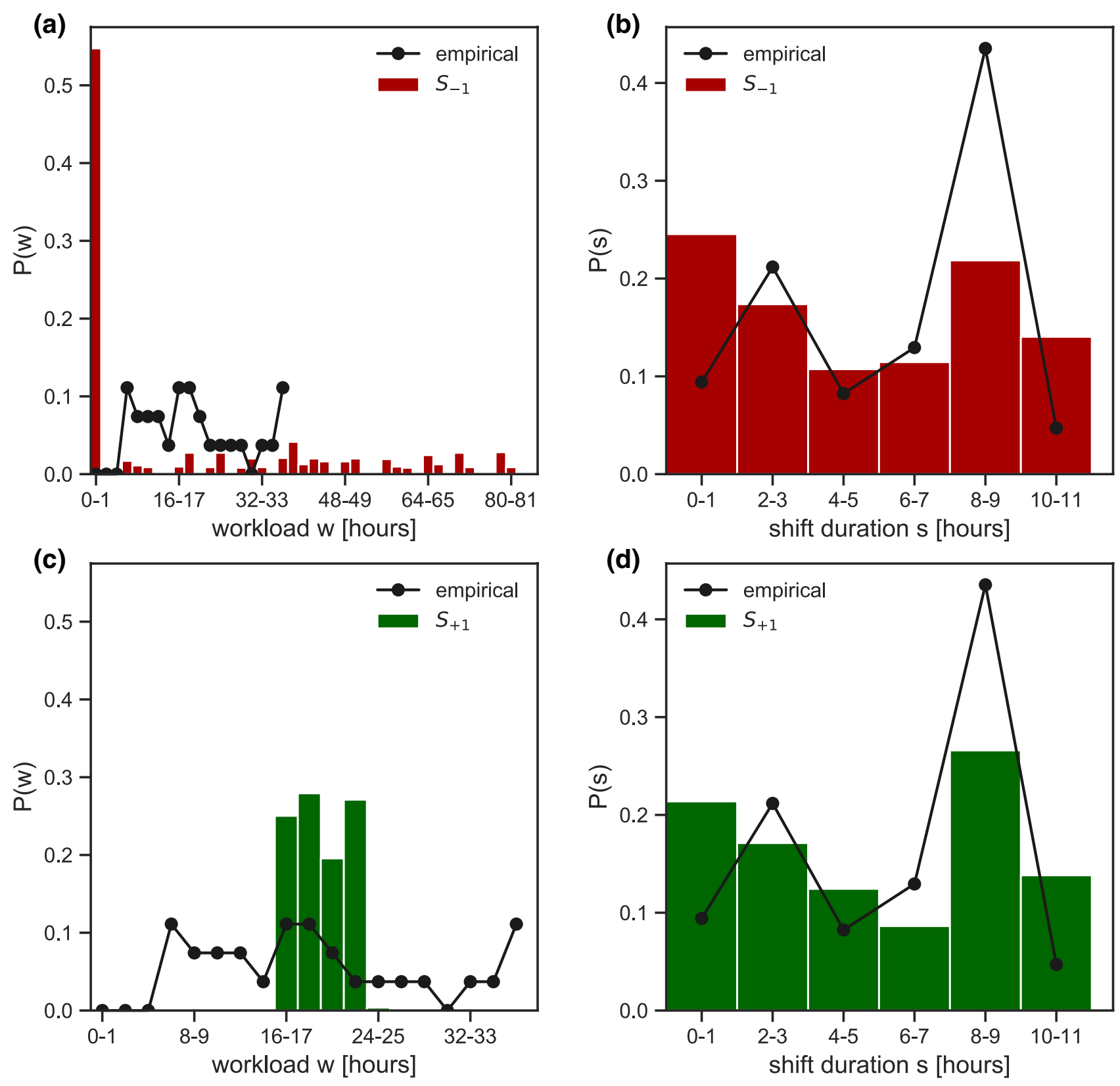

Figure 4. Workload and shift duration in the reorganized schedule under constraints on shift. (a,b) Probability distribution of nurses' workload $w(\mathbf{a})$ and shift duration $s(\mathbf{b})$ following the reorganization of shifts, compared to the empirical distributions. The reorganization is based on model $S_{-1}$ (i.e. constraint on shift duration and attractive potential). (c,d) As panels (b) and (c) for model $S_{+1}$ (constraint on shift duration with repulsive potential).

impact on risk reduction could be achieved by limiting the interactions viable for transmission. Uninformed removal of contacts would be however rather disruptive, with one third of contacts deleted among nurses, at the expense of standards of care. Patient isolation, staff cohorting, and increase in staff levels were shown in previous work to be effective in limiting transmission, by directly or indirectly acting on the interactions ${ }^{18,23,29}$. Fully isolating about $30 \%$ of patients in the ward, however, had no impact on the risk of transmission in this study. Also, the highest improvement in infection control $\left(S_{+1}\right)$ was due neither to cohorting nor to an increase in staff levels. However, this intervention led to the strongest reduction in the fluctuations in the number of distinct contacts per nurse. Homogenizing nurses' contact patterns around 40-45 contacts per nurse removes the presence of potential superspreaders ${ }^{11,12,19,20}$ that could otherwise act as risk amplifiers. The reduction in the degree fluctuations is indeed observed only in the models reducing HAI risk.

Adequate staffing levels and reasonable workloads are established factors promoting infection prevention in hospitals ${ }^{12,18,34}$. Reorganizing nurses' shifts just respecting the maximum shift duration constraint (model $S_{-1}$ ) results in our study in approximately half of the staff not working, while nursing care is assigned to the remaining half, thus forcing unrealistic work schedules (up to $80 \mathrm{~h}$ of work per nurse in 4 days, i.e. an average of $20 \mathrm{~h}$ / 

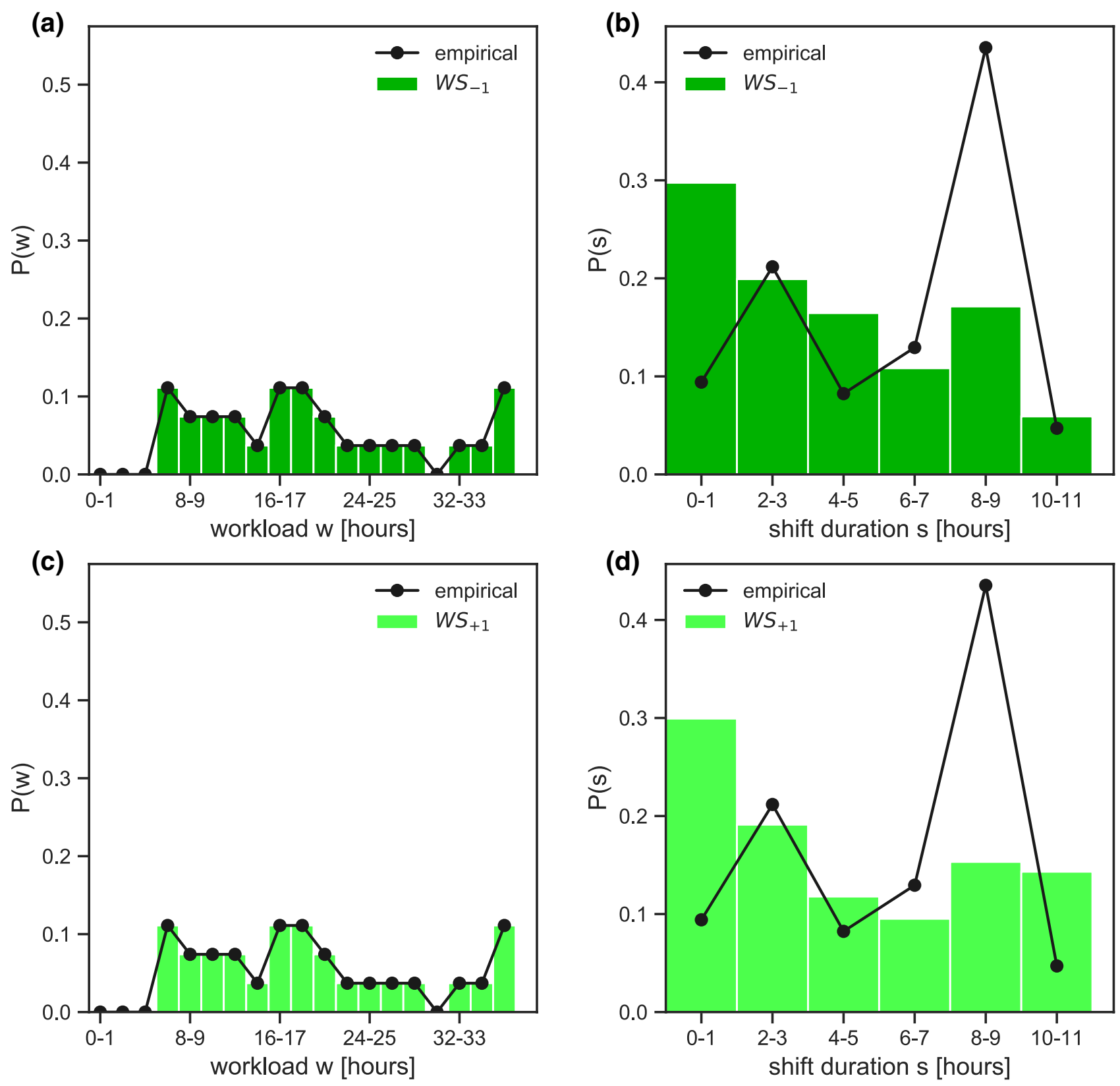

Figure 5. Workload and shift duration in the reorganized schedule under constraints on shift and workload. $(\mathbf{a}, \mathbf{b})$ Probability distribution of nurses' workload $w(\mathbf{a})$ and shift duration $s(\mathbf{b})$ following the reorganization of shifts, compared to the empirical distributions. The reorganization is based on model $W S_{-1}$ (i.e. constraint on workload and shift duration and attractive potential). (c,d): As panels $(\mathbf{b})$ and $(\mathbf{c})$ for model $W S_{+1}$ (constraint on workload and shift duration with repulsive potential).

day). Such reorganization of work has a negative impact on infection control, in line with empirical findings that recognize high workloads, understaffing, and the presence of superspreaders as key risk factors for MRSA circulation in healthcare settings ${ }^{12,34}$. In addition, poor infection control would be here associated to an increase of cohorting levels, which simply results from lower staffing. All other models lead instead to an important reduction of HAI risk, with the reorganization being able to break potential chains of transmission through the swapping of tasks. Improved control is achieved by reducing the presence of superspreaders in the ward, under both regular and irregular individual work timelines of nurses, and with different shift and workload distributions. These findings uncover the practical mechanism for improved control and highlights the robustness of the proposed strategy to different requirements on the organization of the workforce. Crucially, avoiding the presence of nurses with a high number of contacts emerges as more effective in reduction of HAI risk, than reducing the number of nurses assigned to each patient.

Mathematical models have already been used to improve our understanding of hospital epidemiology ${ }^{35,36}$. They are nowadays increasingly data-driven thanks to remote sensing, allowing an automated collection of close-proximity interactions between individuals, not affected by reporting or observer biases inherent to other 

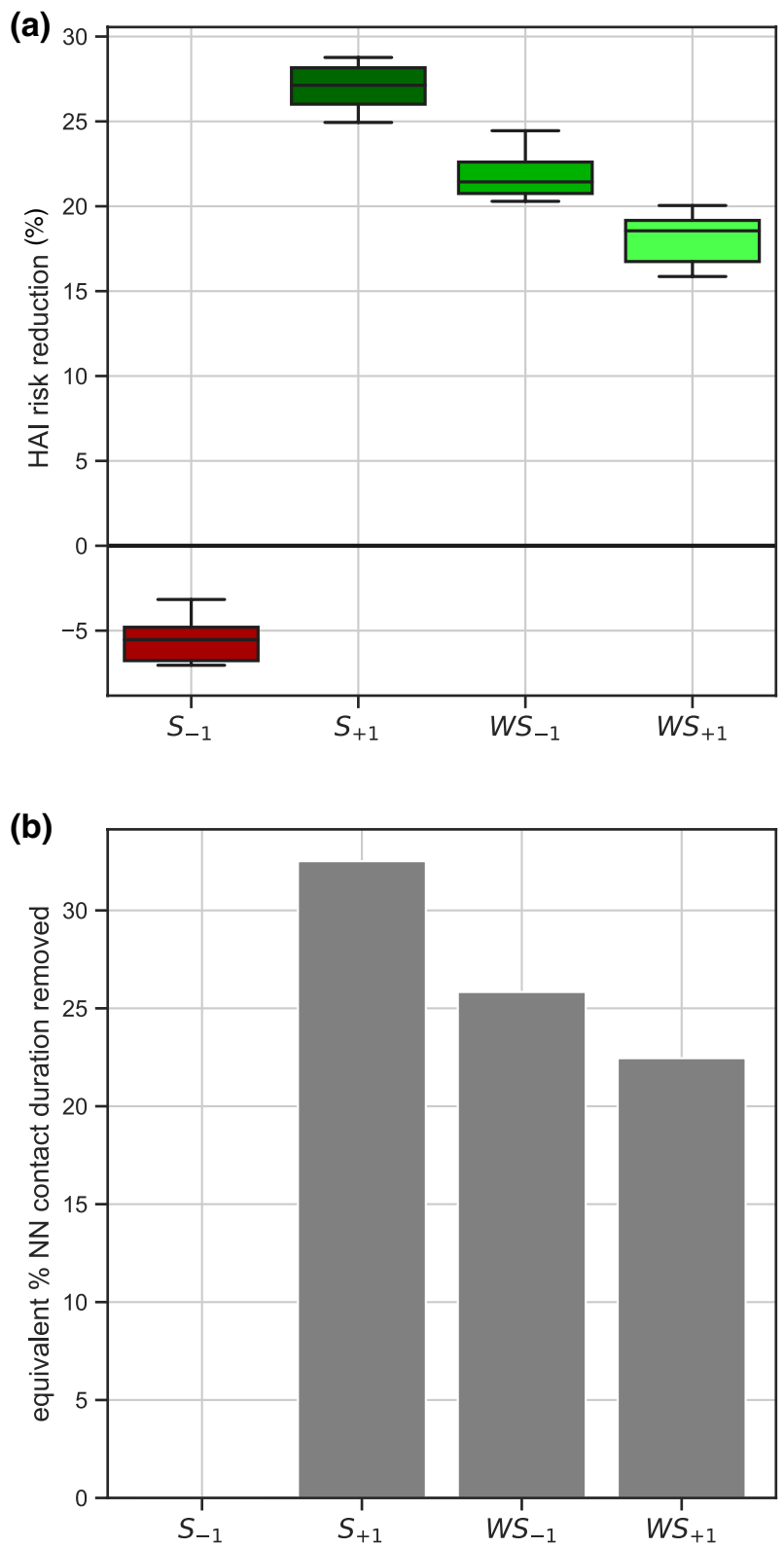

Figure 6. Impact of intervention through reorganization of nurse scheduling. (a) HAI risk reduction in the hospital ward achieved with the reorganization of nurse scheduling in the models $S_{-1}, S_{+1}, W S_{-1}, W S_{+1}$, compared to the empirical situation. Boxplots indicate the median, interquartile range and $95 \% \mathrm{CI}$ of the risk reduction, accounting for the stochasticity of the exchange (results from 50 random trials). (b) Percentage of contact duration to be removed in the nurse-nurse interactions so that the intervention through contact removal would achieve the same risk reductions of panel (a) (obtained through the reorganization of nurse scheduling). $S_{-1}$ is not shown as it has a negative impact on the risk.

approaches $^{37}$. This type of contacts was recently shown to explain the diffusion path of several HAIs ${ }^{6,7,10,38}$. For this reason, our findings extend to pathogens other than MRSA and VRE, spreading along the same routes, under the hypothesis of relatively rapid decolonization ${ }^{6,19}$. Durations of the order of months that are empirically observed in absence of interventions ${ }^{39}$ were not examined here because considered inappropriate in the hypothesis of decontamination taking place.

Prior modeling work generally relied on numerical simulations of HAI spread ${ }^{35}$. We used the infection propagator approach to estimate HAI risk reduction in a reliable and computationally fast way. This approach was already used to estimate the risk of disease persistence in other epidemic contexts ${ }^{32,33}$, and has the advantage of being flexible to the integration of heterogeneities in the force of infection that may depend, for example, on class-specific transmissibility.

The proposed reorganization of staff schedules focused on the class of nurses, as theoretical results on isolation or contact removal clearly identified nurses as the category of healthcare workers who mostly contribute to 

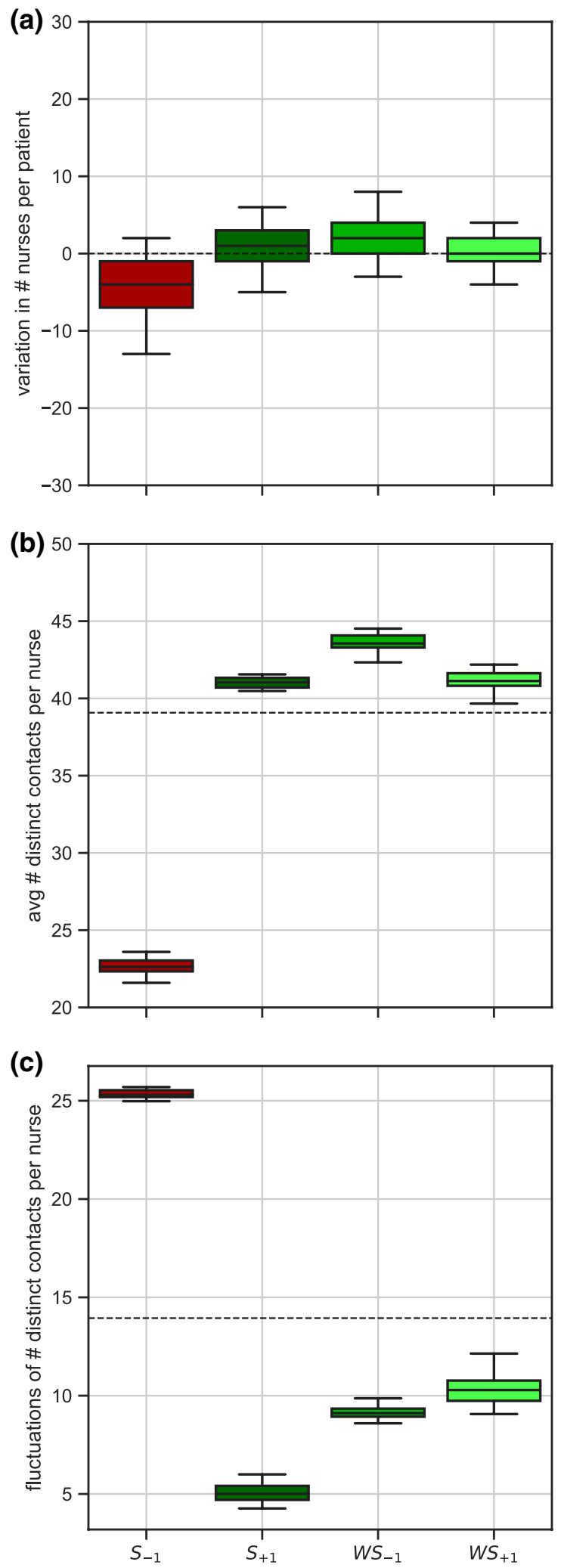

Figure 7. Effect of reorganization on contact patterns. (a) Variation of the number of distinct nurses assigned to each patient, in the reorganized vs. empirical contact pattern. (b) Average number of distinct contacts per nurse (nurses' degree). Dashed line corresponds to the empirical value. (c) Fluctuations (standard deviation) of the number of distinct contacts per nurse. Dashed line corresponds to the empirical value. For $(\mathbf{a}-\mathbf{c})$, results are obtained from 500 random trials. 
the transmission risk in the ward. This can be traced back to the larger number and longer duration of contacts established by nurses, commonly required by nursing care, and it is in line with previous observations and modeling works proposing nurses as target group for prevention measures ${ }^{7,9,22}$. Notwithstanding, further studies may explore the possibility of shift rescheduling for physicians, and other medical staff. Their contacts with patients may be fewer and shorter, but compliance to hygiene measures may depend on the role and task. Differential risk associated with different healthcare professionals and types of contacts could be included in the methodology, including data on hand hygiene practices ${ }^{17}$.

Our findings show the potential to integrate infection prevention into the nurse scheduling problem, originally designed to optimize hospital workforce. However, some steps are still needed to carry this novel paradigm into practice, in the form of a roadmap to a future hospital protocol. First, contact data used in our study were collected in 2013, and cover a specific hospital ward, over a short timeframe. The data are similar to the few other studies available in hospitals regarding the number and nature of contacts of health care workers ${ }^{40}$. This illustrates the generality over time and space of hospital operations. However, there are also features specific to short term vs long term care facilities, prompting the interest of deploying data collection in other contexts. Our methodology could however readily be adapted to prospective schedule planning in other locations. In addition, following the current health crisis, collecting again these data will determine whether the COVID-19 pandemic has brought permanent changes in the organization of hospital operations. Moreover, collecting metadata on the type of clinical interventions performed in the ward, the specific roles of subclasses of personnel (e.g. nurse types), the type of patients admitted, and the standard and organization of care (e.g. scheduling practices along the $24 \mathrm{~h}$ ) is key to improve the parameterization of the epidemic transmission model and define the conditions for task' reassignments (e.g. by swapping similar tasks, or tasks that can be handled by the same staff type). Most importantly, such additional data will help constrain the potential function to patient needs and staff requirements. We list, for example, the length of shifts, the number of weekends worked, the number of on/off days, the role of additional personnel (e.g. physical therapists, nutritionists, with different working patterns), the use of part-time and temporary nursing personnel (thus introducing staff for substitute shifts). Operationally, individual work constraints (e.g., role, days off) can be added to our algorithm using the same methodology used for shift duration and workload. Constraints on specific patient needs can be included by classifying patients according to their necessities, and allowing reassignments of tasks involving patients with similar profiles. A way to implement this is to define, for each nurse, a vector v_c that encodes the fraction of time spent in contacts with patients each class $c$, over the total contact time, for a given set of patient classes (c). Pairwise similarity metrics between vectors (e.g., cosine similarity) could then measure how similar the work profiles of two nurses are. This could then turn into a hard constraint (i.e., allowing changes only between nurses whose vector similarity is above a certain threshold), or a soft constraint (i.e., probability of change increases with vector similarity). Integrating all these elements would make the re-scheduling feasible, without altering the core of the strategy proposed here.

\section{Conclusions}

We presented modeling evidence that reorganizing nurse scheduling while maintaining the number, timeliness, and quality of medical services provided by nursing staff can strongly decrease the risk for HAI diffusion in the hospital ward. Our study provides the theoretical basis for a new control paradigm, showing its potential for integration in future nurse scheduling practices for the implementation of successful infection control programs at the hospitals.

\section{Data availability}

The data used are publicly available and can be found at www.sociopatterns.org.

Received: 8 July 2020; Accepted: 18 March 2021

Published online: 01 April 2021

\section{References}

1. OECD. Healthcare-associated infections. 142-143 (2016) https://doi.org/10.1787/health_glance_eur-2016-46-en.

2. Cohen, M. L. Epidemiology of drug resistance: Implications for a post-antimicrobial era. Science 257, 1050-1055 (1992).

3. Vanhems, P. et al. Estimating potential infection transmission routes in hospital wards using wearable proximity sensors. PLoS ONE 8, e73970 (2013).

4. Lowery-North, D. W. et al. Measuring social contacts in the emergency department. PLoS ONE 8, e70854 (2013).

5. Hertzberg, V. S. et al. Contact networks in the emergency department: Effects of time, environment, patient characteristics, and staff role. Soc. Netw. C, 181-191 (2017).

6. Obadia, T. et al. Detailed contact data and the dissemination of Staphylococcus aureus in hospitals. PLoS Comput. Biol. 11, e1004170 (2015).

7. Voirin, N. et al. Combining high-resolution contact data with virological data to investigate influenza transmission in a tertiary care hospital. Infect. Control Hosp. Epidemiol. 36, 254-260 (2015).

8. Hornbeck, T. et al. Using sensor networks to study the effect of peripatetic healthcare workers on the spread of hospital-associated infections. J. Infect. Dis. 206, 1549-1557 (2012).

9. Bernard, H., Fischer, R., Mikolajczyk, R. T., Kretzschmar, M. \& Wildner, M. Nurses' contacts and potential for infectious disease transmission. Emerg. Infect. Dis. 15, 1438-1444 (2009).

10. Obadia, T. et al. Interindividual contacts and carriage of methicillin-resistant Staphylococcus aureus: A nested case-control study. Infect. Control Hosp. Epidemiol. 36, 922-929 (2015).

11. Meyers, L. A., Pourbohloul, B., Newman, M. E. J., Skowronski, D. M. \& Brunham, R. C. Network theory and SARS: Predicting outbreak diversity. J. Theor. Biol. 232, 71-81 (2005).

12. Albrich, W. C. \& Harbarth, S. Health-care workers: Source, vector, or victim of MRSA?. Lancet Infect. Dis. 8, 289-301 (2008).

13. Cookson, B. et al. Staff carriage of epidemic methicillin-resistant Staphylococcus aureus. J. Clin. Microbiol. 27, 1471-1476 (1989). 
14. Blok, H. E. M. et al. Role of healthcare workers in outbreaks of methicillin-resistant Staphylococcus aureus: A 10-year evaluation from a Dutch University Hospital. Infect. Control Hosp. Epidemiol. 24, 679-685 (2003).

15. Pittet, D. et al. Evidence-based model for hand transmission during patient care and the role of improved practices. Lancet Infect. Dis. 6, 641-652 (2006).

16. Pittet, D. Compliance with handwashing in a teaching hospital. Ann. Intern. Med. 130, 126 (1999).

17. Mastrandrea, R., Soto-Aladro, A., Brouqui, P. \& Barrat, A. Enhancing the evaluation of pathogen transmission risk in a hospital by merging hand-hygiene compliance and contact data: A proof-of-concept study. BMC Res. Notes 8, 426 (2015).

18. Grundmann, H., Hori, S., Winter, B., Tami, A. \& Austin, D. J. Risk factors for the transmission of methicillin-resistant Staphylococcus aureus in an adult intensive care unit: Fitting a model to the data. J. Infect. Dis. 185, 481-488 (2002).

19. Temime, L. et al. Peripatetic health-care workers as potential superspreaders. Proc. Natl. Acad. Sci. 106, 18420-18425 (2009).

20. Lloyd-Smith, J. O., Schreiber, S. J., Kopp, P. E. \& Getz, W. M. Superspreading and the effect of individual variation on disease emergence. Nature 438, 355 (2005).

21. Roberge, R. J. Face shields for infection control: A review. J. Occup. Environ. Hyg. 13, 235-242 (2016).

22. Polgreen, P. M., Tassier, T. L., Pemmaraju, S. V. \& Segre, A. M. Prioritizing healthcare worker vaccinations on the basis of social network analysis. Infect. Control Hosp. Epidemiol. 31, 893-900 (2010).

23. Cooper, B. S. et al. Isolation measures in the hospital management of methicillin resistant Staphylococcus aureus (MRSA): systematic review of the literature. BMJ 329, 533 (2004).

24. McLaws, M.-L. The relationship between hand hygiene and health care-associated infection: It's complicated. Infect. Drug Resist. 8, 7-18 (2015)

25. Siferd, S. P. \& Benton, W. C. Workforce staffing and scheduling: Hospital nursing specific models. Eur. J. Oper. Res. 60, 233-246 (1992).

26. Burke, E. K., De Causmaecker, P., Berghe, G. V. \& Van Landeghem, H. The state of the art of nurse rostering. J. Sched. 7, 441-499 (2004).

27. SocioPatterns.org. http://www.sociopatterns.org/.

28. Holme, P. \& Saramäki, J. Temporal networks. Phys. Rep. 519, 97-125 (2012).

29. Ueno, T. \& Masuda, N. Controlling nosocomial infection based on structure of hospital social networks. J. Theor. Biol. 254, 655-666 (2008).

30. Stehlé, J. et al. Simulation of an SEIR infectious disease model on the dynamic contact network of conference attendees. BMC Med. 9, $87(2011)$

31. Valdano, E., Ferreri, L., Poletto, C. \& Colizza, V. Analytical computation of the epidemic threshold on temporal networks. Phys. Rev. X 5, 021005 (2015).

32. Valdano, E., Poletto, C. \& Colizza, V. Infection propagator approach to compute epidemic thresholds on temporal networks: Impact of immunity and of limited temporal resolution. Eur. Phys. J. B 88, (2015).

33. Darbon, A. et al. Disease persistence on temporal contact networks accounting for heterogeneous infectious periods. R. Soc. Open Sci. 6, 181404 (2019).

34. Vicca, A. F. Nursing staff workload as a determinant of methicillin-resistant Staphylococcus aureus spread in an adult intensive therapy unit. J. Hosp. Infect. 43, 109-113 (1999).

35. Bonten, M. J., Austin, D. J. \& Lipsitch, M. Understanding the spread of antibiotic resistant pathogens in hospitals: Mathematical models as tools for control. Clin. Infect. Dis. Off. Publ. Infect. Dis. Soc. Am. 33, 1739-1746 (2001).

36. Assab, R. et al. Mathematical models of infection transmission in healthcare settings: Recent advances from the use of network structured data. Curr. Opin. Infect. Dis. 30, 410-418 (2017).

37. Mastrandrea, R., Fournet, J. \& Barrat, A. Contact patterns in a high school: A comparison between data collected using wearable sensors, contact diaries and friendship surveys. PLoS ONE 10, e0136497 (2015).

38. Duval, A. et al. Close proximity interactions support transmission of ESBL-K. pneumoniae but not ESBL-E. coli in healthcare settings. PLoS Comput. Biol. 15, e1006496 (2019).

39. Marschall, J. \& Mühlemann, K. Duration of methicillin-resistant Staphylococcus aureus carriage, according to risk factors for acquisition. Infect. Control Hosp. Epidemiol. 27, 1206-1212 (2006).

40. Duval, A. et al. Measuring dynamic social contacts in a rehabilitation hospital: Effect of wards, patient and staff characteristics. Sci. Rep. 8, $1686(2018)$

\section{Acknowledgements}

We acknowledge the Scientific Evolutionary Writing workshop (www.sew-workshop.org) where part of this paper was written.

\section{Author contributions}

E.V. and V.C. conceived and designed the study. E.V. carried out the calculations and the simulations. All authors interpreted the results. V.C. wrote the manuscript. All authors reviewed the manuscript.

\section{Funding}

This study was partially supported by the French ANR project SPHINX (ANR-17-CE36-0008-05) to VC.

\section{Competing interests}

The authors declare no competing interests.

\section{Additional information}

Supplementary Information The online version contains supplementary material available at https://doi.org/ 10.1038/s41598-021-86637-w.

Correspondence and requests for materials should be addressed to V.C.

Reprints and permissions information is available at www.nature.com/reprints.

Publisher's note Springer Nature remains neutral with regard to jurisdictional claims in published maps and institutional affiliations. 
(c) (i) Open Access This article is licensed under a Creative Commons Attribution 4.0 International cc) License, which permits use, sharing, adaptation, distribution and reproduction in any medium or format, as long as you give appropriate credit to the original author(s) and the source, provide a link to the Creative Commons licence, and indicate if changes were made. The images or other third party material in this article are included in the article's Creative Commons licence, unless indicated otherwise in a credit line to the material. If material is not included in the article's Creative Commons licence and your intended use is not permitted by statutory regulation or exceeds the permitted use, you will need to obtain permission directly from the copyright holder. To view a copy of this licence, visit http://creativecommons.org/licenses/by/4.0/.

(C) The Author(s) 2021 